\title{
Envelhecimento e deficiência auditiva referida: um estudo de base populacional
}

\author{
Aging and self-reported hearing loss: \\ a population-based study
}

\footnotetext{
1 Faculdade de Saúde Pública, Universidade de São Paulo, São Paulo, Brasil. 2 Secretaria Municipal da Saúde de São Paulo, São Paulo, Brasil.

3 Faculdade de Ciências Médicas, Universidade Estadual de Campinas, Campinas, Brasil.

4 Faculdade de Medicina de Botucatu, Universidade Estadual Paulista Júlio de Mesquita Filho, Botucatu, Brasil.

5 Faculdade de Medicina, Universidade de São Paulo São Paulo, Brasil.

Correspondência K. M. Paiva

Faculdade de Saúde Pública Universidade de São Paulo. Rua Oscar Freire 2121, apto. 1402, São Paulo, SP 05409-011, Brasil. kmpaiva@usp.br
}

\section{Abstract}

The aim of this study was to estimate the prevalence of self-reported hearing loss among elderly people in São Paulo, Brazil, according to sociodemographic characteristics and to identify associated factors. Data were from the São Paulo Health Survey (ISA-Capital 2003), and the subgroup of elderly $(n=872)$ was analyzed. The $\chi^{2}$ test was used to verify the association between self-reported hearing loss and socio-demographic characteristics. The statistical analyses used Poisson regression. Prevalence of self-reported hearing loss in the elderly was $11.2 \%$, and was higher in men ( $P R=1.86$; 95\%CI: 1.19-2.92). There was an important lack of knowledge among the elderly regarding the causes of their hearing loss (42.5\%). Among the sample, $25.5 \%$ reported difficulties in leisure-time activities and $11.4 \%$ needed help in routine activities, while $63.3 \%$ required no assistance due to their impairment. The high prevalence of self-reported hearing loss in the elderly, especially in men, highlights the importance of this public health problem. Population aging is a new reality, placing increasing demands on healthcare professionals and government.

Aging; Hearing Loss; Aged; Prevalence
Karina Mary de Paiva 1

Chester Luis Galvão Cesar 1

Maria Cecília Goi Porto Alves 2

Marilisa Berti de Azevedo Barros 3

Luana Carandina 4

Moises Goldbaum 5

\section{Introdução}

O envelhecimento envolve alterações na saúde geral do indivíduo, que podem resultar em comprometimento de funções fisiológicas, imunológicas e sensoriais, como é o caso da audição. A perda auditiva no idoso pode ocorrer de forma progressiva, específica e ter caráter individual, sendo denominada presbiacusia, e suas complicações podem representar consequências sociais e psicológicas, como o isolamento social, frustração e depressão 1,2,3.

A prevalência da deficiência auditiva em idosos é alta 4,5,6,7,8. Entretanto, a falta de estudos de base populacional em âmbito nacional, pode explicar o fato de a Política Nacional de Saúde da Pessoa Portadora de Deficiência fazer referência à literatura internacional, ao citar a presbiacusia como principal causa de deficiência auditiva em idosos, com incidência de aproximadamente $30 \%$ na população com mais de 65 anos 9 .

A avaliação audiológica na pessoa idosa deve envolver não só os exames que visam definir os limiares audiológicos do indivíduo, como a audiometria tonal, mas também considerar a percepção do paciente em relação a sua perda auditiva, no aspecto funcional, ou seja, nas suas atividades sociais, familiares e diárias 10,11.

Embora o exame de audiometria possa ser considerado padrão ouro para estimar a prevalência da deficiência auditiva em uma po- 
pulação, questões como orçamento limitado e dificuldades logísticas têm impossibilitado a realização desses exames em projetos de base populacional. Assim, estudos internacionais compararam os resultados de testes audiométricos e questionários de autoavaliação para percepção da deficiência auditiva e encontraram prevalências similares dimensionadas pelos questionários de autopercepção e medidas de perda auditiva 2,12,13,14. Hashimoto et al. 14 relataram uma concordância entre o autorrelato de perda auditiva e a detecção de perda auditiva pela audiometria tonal de $83 \%$ a partir dos 60 anos. Eles concluíram que a avaliação subjetiva da deficiência auditiva não deve representar um critério de substituição do exame de audiometria, mas pode ser utilizada como instrumento de triagem auditiva.

O Programa de Prevenção da Cegueira e da Surdez da Organização Mundial da Saúde (OMS), tem se empenhado na criação de um banco de dados sobre deficiência auditiva a partir de uma única pergunta referente a como o indivíduo percebe sua audição. A finalidade deste é determinar a extensão da carga global desta incapacidade, assim como gerar dados recentes e comparáveis do ponto de vista epidemiológico. Estudos de base populacional se tornam essenciais neste contexto na medida em que possibilitam a geração de importantes indicadores de comparabilidade em nível internacional e permitem a avaliação de prioridades e intervenções em nível populacional 15 .

A prevalência da deficiência auditiva referida por idosos no Município de São Paulo representa um relevante indicador tanto para os profissionais da saúde quanto para o poder público. Estes dados podem auxiliar no direcionamento de investimentos em educação continuada das equipes de saúde, na garantia dos direitos dos idosos e na criação de condições para promover autonomia, integração e participação efetiva na sociedade. Questões referentes ao envelhecimento populacional têm adquirido especial importância devido ao aumento do número de idosos na população, principalmente nos países em desenvolvimento. O ritmo acelerado deste aumento tende a dificultar a adaptação e a adequação necessárias para atender as demandas desta parcela vulnerável da população.

O objetivo deste estudo é estimar a prevalência da deficiência auditiva referida em idosos do Município de São Paulo segundo características sociodemográficas e descrever as características atribuídas a essa deficiência.

\section{Metodologia}

Foi utilizado o banco de dados do Inquérito de Saúde no Município de São Paulo - ISA-Capital 2003, estudo transversal de base populacional que envolveu a população não-institucionalizada e residente em área urbana do Município de São Paulo no ano de 2003, e contou com o financiamento da Secretaria Municipal de Saúde do Estado de São Paulo (SMS-SP). O inquérito teve como objetivo analisar as condições de vida e de saúde da população, além do uso de serviços de saúde no Município de São Paulo.

A coleta de dados foi realizada por meio de entrevistas domiciliares com aplicação do questionário por entrevistadores previamente treinados, avaliados e reciclados durante todo o período da pesquisa. A elaboração do questionário foi baseada nos objetivos do inquérito e foram criados blocos organizados por conjuntos temáticos e composto por questões fechadas, abertas e semi-abertas 16,17 .

A amostra do ISA-Capital (2003) foi probabilística e utilizou-se o método de amostragem estratificada por conglomerado em dois estágios. No primeiro estágio foram sorteados os setores censitários e, posteriormente, dentro de cada setor, os domicílios. Os estratos foram construídos pelo agrupamento dos setores censitários em três grupos, definidos pelo percentual de chefes de família com nível universitário. Para garantir a representatividade de subgrupos populacionais de interesse, foram definidos oito domínios de estudo (menores de 1 ano, 1-11 anos, mulheres de 12-19 anos, 20-59 anos e 60 anos e mais e homens nas mesmas faixas etárias definidas) 16,17. A população deste estudo envolveu os indivíduos com 60 anos e mais que foram entrevistados no ISA-Capital (2003), totalizando 872 idosos.

A deficiência auditiva referida nesta população foi analisada a partir do bloco de deficiências físicas (E), que engloba questões relacionadas à dificuldade em ouvir, surdez em um dos ouvidos e nos dois ouvidos, tendo sido criada a variável "deficiência auditiva referida" a partir do agrupamento das respostas positivas a estes questionamentos.

Além da prevalência desta variável, foram obtidas estimativas referentes às características sociodemográficas dos idosos: sexo, idade (em faixas etárias), escolaridade do entrevistado (em anos de estudo), situação conjugal (com companheiro e sem companheiro) e renda per capita (em salários mínimos).

Testou-se a associação entre as variáveis e a referência de deficiência auditiva por meio do teste $\chi^{2}$ com nível de $5 \%$ de significância. Foram utilizadas razões de prevalência e intervalos de 
95\% de confiança (IC95\%) e a análise foi conduzida por meio da regressão de Poisson.

Os idosos que referiram deficiência auditiva, responderam a um bloco específico do questionário sobre questões referentes a causas (doenças/causa genética, acidentes de trabalho, idade avançada, ou seja, presbiacusia), necessidade de assistência, médico-hospitalar ou para reabilitação, dificuldades em atividades de lazer e necessidade de ajuda para atividades cotidianas (realização de compras, pagamento de contas, ida ao banco, limpeza da casa e preparo de alimentos. Esses dados foram descritos segundo sexo e foi realizado o teste do $\chi^{2}$ para verificar associação entre características atribuídas à deficiência auditiva e sexo do entrevistado.

O programa utilizado para as análises estatísticas foi o Stata 10 for Windows (Stata Corp., College Station, Estados Unidos), que permite incorporar os pesos decorrentes do delineamento complexo da amostra.

\section{Resultados}

Foram entrevistados 872 idosos com idade média de 70,2 anos (IC95\%: 69,68-70,78). A população feminina foi predominante $(60,3 \%)$ e a maior parte deles pertencia à faixa etária mais jovem, de 60-69 anos (51,1\%). Os mais idosos, ou seja, aqueles que relataram 80 anos e mais representaram 12,3\% dos idosos entrevistados. Quanto à escolaridade e renda, as maiores frequências referiram-se a idosos com oito anos e mais de estudo (39\%) e com renda per capita de um a três salários mínimos $(45,3 \%)$. Com relação à situação conjugal a maioria $(64,1 \%)$ tem companheiro(a) (Tabela 1).

Os dois grupos de idosos segundo referência de deficiência auditiva se distribuíram da seguinte forma: entre os que relataram ter deficiência auditiva, 46,6\% tinham entre 60-69 anos e $16,1 \%$ tinham 80 anos e mais. Para os que não referiram 51,6\% tinham entre $60-69$ anos, $11,8 \%$ a partir de 80 anos. Com relação ao sexo, 44,9\% e $62,2 \%$, respectivamente dos que referiram e

Tabela 1

Características sociodemográficas da população de idosos segundo deficiência auditiva referida. Município de São Paulo, Brasil, $2003(n=872)$

\begin{tabular}{|c|c|c|c|c|c|c|}
\hline \multirow[t]{3}{*}{ Características sociodemográficas } & \multicolumn{4}{|c|}{ Deficiência auditiva referida } & \multicolumn{2}{|c|}{ População de idosos } \\
\hline & \multicolumn{2}{|c|}{ Sim } & \multicolumn{2}{|c|}{ Não } & \multirow[b]{2}{*}{$\%$ * } & \multirow[b]{2}{*}{$\mathrm{n} * *$} \\
\hline & $\%$ * & $\mathrm{n} * \star$ & $\%$ * & $\mathrm{n} * \star$ & & \\
\hline \multicolumn{7}{|l|}{ Sexo } \\
\hline Feminino & 44,9 & 33 & 62,2 & 418 & 60,3 & 451 \\
\hline Masculino & 55,1 & 58 & 37,8 & 363 & 39,7 & 421 \\
\hline \multicolumn{7}{|l|}{ Faixa etária (anos) } \\
\hline $60-69$ & 46,6 & 42 & 51,6 & 432 & 51,1 & 474 \\
\hline $70-79$ & 37,3 & 35 & 36,6 & 272 & 36,6 & 307 \\
\hline 80 e mais & 16,1 & 14 & 11,8 & 77 & 12,3 & 91 \\
\hline \multicolumn{7}{|l|}{ Anos de estudo } \\
\hline Até 3 & 23,3 & 29 & 26,4 & 272 & 26,0 & 301 \\
\hline $4-7$ & 29,1 & 28 & 35,8 & 291 & 35,1 & 319 \\
\hline 8 e mais & 47,6 & 34 & 37,8 & 218 & 38,9 & 252 \\
\hline \multicolumn{7}{|l|}{ Situação conjugal } \\
\hline Com companheiro & 71,6 & 67 & 63,2 & 511 & 64,1 & 578 \\
\hline Sem companheiro & 28,4 & 24 & 36,8 & 279 & 35,9 & 294 \\
\hline \multicolumn{7}{|l|}{ 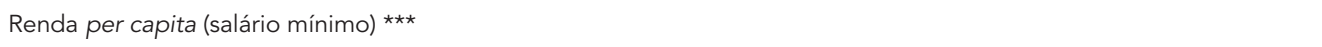 } \\
\hline$<1$ & 38,1 & 40 & 34,8 & 321 & 35,1 & 361 \\
\hline $1-3$ & 40,9 & 39 & 45,9 & 365 & 45,3 & 404 \\
\hline 4 e mais & 21,0 & 12 & 19,3 & 95 & 19,6 & 107 \\
\hline
\end{tabular}


não referiram deficiência, foram mulheres. A maioria relatou ter mais oito anos de estudo tanto para quem referiu quanto para quem não referiu tal deficiência $(47,6 \% ; 37,8 \%)$, ter companheiro $(71,6 \% ; 63,2 \%)$ e ter renda familiar per capita de 1-3 salários mínimos (40,9\%; 45,9\%) (Tabela 1).

A prevalência estimada da deficiência auditiva referida pelos idosos foi de 11,2\% (IC95\%: $8,9-14,0)$, composta por dificuldades de ouvir $(10,1 \%)$, surdez de um ouvido $(1,2 \%)$ e surdez nos dois ouvidos $(0,2 \%)$, correspondentes respectivamente à referência de 78, 11 e 2 idosos (Tabela 2).

A deficiência auditiva referida por sexo foi significativamente mais prevalente nos homens $(\mathrm{RP}=1,86$; IC95\%: 1,19-2,92). Quanto às outras características sociodemográficas, como idade, escolaridade, situação conjugal e renda per capita não foram encontradas associações estatisticamente significativas, embora as estimativas pontuais de prevalência desta deficiência tenham aumentado com a idade e tenham sido maior entre os idosos que relataram alta escolaridade (8 anos e mais) (Tabela 2 ).

Com relação às causas atribuídas, observouse que $21,1 \%$ dos idosos não sabiam o que havia provocado a deficiência auditiva e 21,4\% não identificaram como causa nenhuma das opções apresentadas. Dentre as respostas identificadas, a maior proporção foi atribuída à presbiacusia $(27,1 \%)$, seguida por causas genéticas ou doenças $(16,6 \%)$ e acidentes $(13,8 \%)$, incluindo os de trabalho que representaram 11,5\% (Tabela 3).

A maior proporção de desconhecimento quanto às causas foi relatada pelas mulheres $(48,7 \%)$ e elas mencionaram como principal causa as doenças (24,6\%). Para os homens, a presbiacusia foi relatada como principal causa de deficiência (31,3\%). O valor de p, embora superior a 0,05, esteve muito próximo disso (Tabela 3).

Verificou-se que 25,5\% (IC95\%: 15,4-39,1) destes idosos relataram dificuldades para realização de atividades de lazer e 11,4\% (IC95\%: 5,4-22,4) referiram necessidade de ajuda para atividades cotidianas, como fazer compras,

Estimativas de prevalência $(P)$ e razões de prevalência (RP) de deficiência auditiva referida por idosos, segundo características sociodemográficas. Município de São Paulo, Brasil, 2003 ( $n=872$ ).

\begin{tabular}{|c|c|c|c|}
\hline \multirow[t]{2}{*}{ Características sociodemográficas } & \multicolumn{3}{|c|}{ Deficiência auditiva referida } \\
\hline & $n$ * & P (IC95\%) & RP (IC95\%) \\
\hline Total & 91 & $11,2(8,9-14,0)$ & \\
\hline \multicolumn{4}{|l|}{ Sexo } \\
\hline Feminino & 33 & $8,3(5,8-11,7)$ & 1,00 \\
\hline Masculino & 58 & $15,5(11,5-20,5)$ & $1,86(1,19-2,92)$ \\
\hline \multicolumn{4}{|l|}{ Faixa etária (anos) } \\
\hline $60-69$ & 42 & $10,2(7,4-13,8)$ & 1,00 \\
\hline $70-79$ & 35 & $11,3(7,2-13,4)$ & $1,11(0,67-1,84)$ \\
\hline 80 e mais & 14 & $14,6(9,0-22,7)$ & $1,43(0,77-2,67)$ \\
\hline \multicolumn{4}{|l|}{ Anos de estudo } \\
\hline Até 3 & 29 & $10,0(6,6-14,7)$ & 1,00 \\
\hline $4-7$ & 28 & $9,3(5,7-14,8)$ & $0,93(0,46-1,86)$ \\
\hline 8 e mais & 34 & $13,7(8,9-13,9)$ & $1,37(0,81-2,32)$ \\
\hline \multicolumn{4}{|l|}{ Situação conjugal } \\
\hline Com companheiro & 67 & $12,5(9,2-16,6)$ & 1,00 \\
\hline Sem companheiro & 24 & $8,8(5,6-13,6)$ & $1,41(0,78-2,54)$ \\
\hline \multicolumn{4}{|l|}{ Renda per capita (salário mínimo) ** } \\
\hline$<1$ & 40 & $12,1(8,3-17,2)$ & 1,00 \\
\hline $1-3$ & 12 & $10,1(7,0-14,2)$ & $0,83(0,51-1,37)$ \\
\hline 4 e mais & 39 & $12,0(8,9-14,0)$ & $0,99(0,49-1,99)$ \\
\hline
\end{tabular}

IC95\%: intervalo de 95\% de confiança.

* Número de idosos na amostra não ponderada;

** O salário mínimo entre fevereiro de 2003 e janeiro de 2004 era de $\mathrm{R} \$ 240,00$. 
Características atribuídas à deficiência auditiva referida pelos idosos (causas, dificuldades em atividades, necessidade de ajuda e assistência), segundo o sexo. Município de São Paulo, Brasil, 2003 ( $n=91$ ).

\begin{tabular}{|c|c|c|c|c|}
\hline \multirow{2}{*}{$\begin{array}{l}\text { Características atribuídas à deficiência auditiva } \\
\text { referida }\end{array}$} & \multirow[t]{2}{*}{$\%$ * } & \multicolumn{2}{|c|}{ Sexo } & \multirow[t]{2}{*}{ Valor de $p$ ** } \\
\hline & & Masculino & Feminino & \\
\hline Causas & & & & 0,06 \\
\hline Doenças/Genético & 16,6 & 10,0 & 24,6 & \\
\hline Acidentes de trabalho & 13,8 & 21,3 & 4,5 & \\
\hline Idade avançada & 27,1 & 31,3 & 22,1 & \\
\hline Outros/NS/NR & 42,5 & 37,4 & 48,7 & \\
\hline Dificuldades em atividades de lazer & & & & 0,70 \\
\hline Sim & 25,5 & 23,7 & 27,7 & \\
\hline Não & 74,5 & 76,3 & 72,3 & \\
\hline Necessidade de ajuda em atividades cotidianas & & & & 0,08 \\
\hline Sim & 11,4 & 5,5 & 18,5 & \\
\hline Não & 88,6 & 94,5 & 81,5 & \\
\hline Necessidade de assistência & & & & 0,37 \\
\hline $\operatorname{Sim}$ & 36,7 & 42,9 & 29,0 & \\
\hline Não & 63,3 & 57,1 & 71,0 & \\
\hline
\end{tabular}

NR: não respondeu; NS: não sabe.

* Proporção (\%) na amostra de idosos ponderada;

** Teste de associação $\chi^{2}$.

ir ao banco e pagar contas. A maioria dos idosos relatou não necessitar de nenhuma assistência decorrente desta deficiência (63,3\%; IC95\%: 50,2-74,8) (Tabela 3).

Com relação ao sexo, verificou-se que foram as mulheres as que mais apresentaram queixas relativas à necessidade de ajuda para realização de atividades cotidianas (18,5\%), embora as diferenças fossem detectadas com nível de significância igual a 0,08. Para relatos de necessidade de assistência em decorrência da deficiência auditiva, a proporção de homens que disseram precisar de assistência é que foi maior comparada às mulheres (42,9\%; 29\%), embora não significativa estatisticamente $(p=0,37)$.

\section{Discussão}

A escassez de dados de prevalência de deficiência auditiva na população brasileira pode ser atribuída às dificuldades e/ou impossibilidade para realização de exames de audiometria em estudos de base populacional. Pesquisas envolvendo diagnóstico audiológico por meio da detecção de limiares tonais apresentam diferentes prevalências desta deficiência $4,5,6,7,8$. Isto pode ser explicado pela diversidade das metodologias aplicadas, principalmente no que se refere à po- pulação estudada, já que estes estudos envolvem a população que chega aos serviços de saúde.

Vale ressaltar que a deficiência auditiva neste estudo, assim como suas causas e limitações, foi determinada a partir da percepção dos entrevistados e não envolveu nenhuma confirmação diagnóstica. A percepção do idoso quanto à presença de deficiência auditiva pode estar sujeita a uma série de questões que envolvem a experiência de vida, cultura, escolaridade e do contexto em que está inserido cada entrevistado. Assim, atitudes quanto à aceitação consciente e/ou inconsciente da deficiência, assimilação desta como sendo parte do processo do envelhecimento e adaptação às possíveis dificuldades através de reorganização do ambiente, utilização de recursos alternativos e afastamento de situações que possam representar obstáculos à sua comunicação, possivelmente influenciaram o idoso a referir deficiência auditiva. Além disso, a prevalência de morbidades referidas pode ser subestimada, mediante problemas de memória e/ou ausência de diagnóstico 18.

Apesar disso, a validade da autorreferência da deficiência auditiva tem sido avaliada por alguns autores 2,12,13. Torre III et al. 13 observaram que a utilização de uma única pergunta referente à percepção da deficiência auditiva pode representar uma ferramenta de triagem por ser tanto sensível 
(74\%) quanto específica (72\%), superando barreiras culturais e de linguagem.

A deficiência auditiva referida representa uma ferramenta essencial para detecção de indicadores em nível populacional, além de se constituir em uma estratégia para serviços de atenção primária, como ferramenta de triagem auditiva para posterior encaminhamento aos serviços especializados em saúde auditiva.

Nondahl et al. 12 já afirmavam que inquéritos de base populacional constituem um meio relativamente rápido e barato de obter estimativas de prevalência de perda auditiva em grandes populações, bem como em áreas geográficas pequenas e para subgrupos populacionais específicos. Assim, destacavam a necessidade de mais estudos populacionais para idosos, com a finalidade de medir claramente a magnitude deste problema de saúde pública e começar a identificar intervenções em nível primário, secundário e terciário.

A prevalência da deficiência auditiva referida pelos idosos neste estudo foi $11,2 \%$, sendo que os idosos do sexo masculino apresentaram maiores prevalências quando comparados às mulheres.

A maior prevalência da deficiência auditiva no sexo masculino está em consonância com a literatura 19,20,21,22,23,24. De forma semelhante, outros estudos vêm observando um maior decréscimo dos limiares tonais, principalmente nas altas frequências, em idosos do sexo masculino quando comparados às mulheres 13,25,26,27.

A maior prevalência da deficiência auditiva em homens remete à necessidade de investimentos voltados à prevenção. $\mathrm{O}$ diagnóstico da presbiacusia pode não ser uma tarefa fácil, pois o envelhecimento do ouvido humano é resultado de efeitos cumulativos de diversos fatores que podem ser desencadeadores ou potencializadores da perda auditiva. Por desencadeadores podemos citar os fatores intrínsecos: hereditariedade, fatores metabólicos e vasculares; os potencializadores podem envolver fatores extrínsecos, como exposição a ruído ocupacional e não-ocupacional, utilização de medicamentos, nutrição e estresse 28,29. Embora não seja possível intervir nos primeiros, a prevenção quanto aos fatores extrínsecos é possível e são os homens os que estariam mais expostos a estes fatores.

Neste estudo, a prevalência encontrada de deficiência auditiva referida dentre os mais idosos (80 anos e mais) foi de $14,6 \%$ e apesar de ter-se observado uma tendência de maiores prevalências conforme o aumento da idade, estes resultados não foram estatisticamente significativos ( $\mathrm{p}=0,535)$. É importante observar que o número de idosos com deficiência auditiva em cada faixa etária foi pequeno, o que não pode ser descarta- do como responsável pela não detecção das diferenças esperadas. A maior prevalência de deficiência auditiva entre os mais idosos é encontrada na literatura 6,7,19,24. Alguns autores esclarecem que devido ao caráter insidioso e progressivo da presbiacusia, sua prevalência aumenta substancialmente com a idade 30,31 .

A presbiacusia pode ser mascarada nos idosos, pois seu caráter lento pode gerar incapacidades apenas com o avançar da idade, devido ao aumento do tempo de privação auditiva. Esta característica pode facilitar a adaptação inicial do idoso às dificuldades e limitações que por se relacionarem à inteligibilidade de fala, permitem ao idoso mudar sua rotina e evitar situações que possam representar um desafio à sua capacidade auditiva e comunicativa. Neste aspecto, as desvantagens vividas pelos idosos podem se confundir com rótulos que frequentemente são conferidos aos idosos: desatento, disperso, confuso, não colaborador, não comunicativo e senil 28 .

A caracterização da deficiência auditiva pelos idosos que referiram esta deficiência representa uma ferramenta para auxiliar na compreensão do significado conferido a esta incapacidade quando se avalia o conhecimento quanto às causas, às dificuldades e às necessidades decorrentes da presença deste déficit e que os levam a relatar a percepção da mesma em suas vidas. O conhecimento dos idosos quanto à sua deficiência e os critérios relacionados a esta referência podem denotar o impacto desta dificuldade auditiva em seu cotidiano.

Quanto às causas atribuídas, 21,1\% desconhecem a causa da deficiência e $27,1 \%$ atribuíram à idade avançada. Este resultado se assemelha ao encontrado em outro estudo que observou que a causa mais prevalente para a deficiência auditiva no grupo de 60 e 75 anos foi a presbiacusia $(17,7 \%)$, com taxas mais elevadas para o grupo com idade igual ou superior a 75 $\operatorname{anos}(41, \%) 32$

Os relatos de $25,5 \%$ dos idosos com dificuldades em atividades de lazer são relevantes, já que a deficiência auditiva pode levar o idoso a se afastar destes tipos de atividade como forma de evitar situações que exijam de sua capacidade auditiva. Além disso, vale ressaltar que não é possível saber se os idosos participam destas atividades, pois os relatos são relacionados às dificuldades para realização das mesmas. Quanto às atividades cotidianas, 11,4\% dos idosos disseram necessitar de ajuda para realizar tarefas como ir ao banco, pagar contas e fazer compras. $\mathrm{O}$ idoso, muitas vezes, muda sua rotina de maneira inconsciente em função da deficiência e passa a evitar certos tipos de atividades que possam causar desconforto. Por outro lado, os relatos de 
não necessitarem de ajuda para estas atividades pode ser justificado pelo fato de estas circunstâncias possibilitarem ao idoso utilizar de estratégias facilitadoras da comunicação como a leitura labial.

Os idosos relataram não necessitar de assistência decorrente da deficiência auditiva referida, o que sugere desconhecimento quanto às diretrizes da Política Nacional do Idoso 33 que expõe em seu decreto o fornecimento de órteses e próteses necessárias ao idoso como responsabilidade do estado. Esta questão torna-se ainda mais relevante quando se observa que a prevalência de deficiência auditiva é maior entre os homens, apontando para a necessidade do aumento de informações quanto à prevenção aos fatores potencializadores da presbiacusia, como a exposição a ruídos ambientais, ocupacionais e no lazer.

Além da prevenção, o processo de reabilitação auditiva em decorrência da presbiacusia é fundamental e envolve atitudes relacionadas à orientação quanto ao uso dos aparelhos auditivos, benefícios e dificuldades decorrentes deste processo, aconselhamento, acompanhamento e treinamento auditivo ${ }^{34}$. A carga física e psíquica envolvida no processo do envelhecimento aliada às questões fisiológicas relacionadas ao processamento auditivo central, como diminuição da memória, da percepção e da atenção, assim como o declínio visual e a diminuição da destreza manual podem gerar grande desmotivação por parte do idoso e poderão influenciar o sucesso desta etapa. Assim, o acompanhamento efetivo por profissionais habilitados, através da terapia fonoaudiológica, torna-se primordial.

Neste sentido a Política Nacional de Atenção à Saúde Auditiva, instituída em 2004 35, representa um grande avanço, já que disponibiliza consultas otorrinolaringológicas, realização de exames de audiometria e processo de reabilitação auditiva, incluindo a colocação de próteses auditivas.

\section{Considerações finais}

A alta prevalência da deficiência auditiva referida pelos idosos, principalmente do sexo masculino, aliada ao desconhecimento quanto às causas e à necessidade de assistência decorrentes deste déficit, apontam para a relevância deste problema para a saúde pública.

$\mathrm{O}$ aumento do número de idosos na população é uma realidade nova para os países como o Brasil e vem acompanhado de dificuldades para direcionar esforços que atendam de forma integral e igualitária às necessidades e demandas geradas por eles.

O conhecimento das políticas relacionadas à audição ainda se mostra pequeno, pois grande parte dos idosos que referiram deficiência auditiva relatou não saber a causa da mesma e não necessitar de nenhuma assistência decorrente da deficiência referida. Cabe às políticas vigentes, como a Política Nacional do Idoso rever sua efetividade e abrangência de seus decretos e à Política Nacional de Atenção à Saúde Auditiva avaliar seus procedimentos e ações, para que suas diretrizes alcancem também este segmento vulnerável da população, garantindo diagnóstico e reabilitação auditiva.

As questões referentes a esta deficiência ainda fazem parte de um universo centrado nos profissionais que lidam diretamente com este processo. A sociedade e o próprio idoso não parecem estar preparados para lidarem com este tipo de privação sensorial, confirmando de certa forma a ideia de que as limitações vividas pelo deficiente auditivo são decorrentes apenas do processo de senescência. 


\section{Resumo}

O objetivo do estudo foi estimar a prevalência da deficiência auditiva referida por idosos do Município de São Paulo, Brasil, segundo características sociodemográficas e descrever características atribuídas a esta deficiência. Os dados são provenientes do Inquérito de Saúde do Município de São Paulo (ISA-Capital/2003), com análise do subgrupo dos idosos $(n=872)$. Realizaram-se o teste do $\chi^{2}$ e a análise de regressão de Poisson. A prevalência da deficiência auditiva referida pelos idosos foi 11,2\% e foi maior entre os homens $(R P=1,86$; IC95\%: 1,19-2,92). Observou-se grande desconhecimento dos idosos quanto às causas da deficiência auditiva referida (42,5\%), 25,5\% relataram ter dificuldades em atividades de lazer, 11,4\% necessitavam de ajuda para atividades cotidianas e 63,3\% relataram não necessitar de assistência em decorrência deste déficit. A alta prevalência de deficiência auditiva referida pelos idosos, principalmente do sexo masculino, remete à relevância deste problema para a saúde pública, já que o envelhecimento populacional é uma realidade nova e vem acompanhada de exigências ainda desconhecidas por profissionais e pelo poder público.

Envelhecimento; Perda Auditiva; Idoso; Prevalência

\section{Colaboradores}

K. M. Paiva contribuiu na revisão da literatura, análise dos resultados e redação do artigo final. C. L. G. Cesar contribuiu na elaboração do projeto ISA-Capital, coordenação da pesquisa de campo, orientação e revisão final do artigo. L. Carandina, M. Goldbaum e M. B. A. Barros participaram na elaboração do projeto ISA-Capital e revisão final do artigo. M. C. G. P. Alves colaborou na elaboração do projeto ISA-Capital, amostragem populacional e revisão final do artigo.

\section{Agradecimentos}

Às agências de fomento que concederam bolsas de mestrado a K. M. Paiva: o Conselho Nacional de Desenvolvimento Científico e Tecnológico (CNPq), processo no ${ }^{\circ}$ 136558/2008-9 e à Fundação de Amparo à Pesquisa do Estado de São Paulo (FAPESP), processo no. 08/52069-6. À Secretaria Municipal de Saúde do Estado de São Paulo pelo financiamento para execução do Inquérito de Saúde do Município de São Paulo (ISACapital-2003).

\section{Referências}

1. Gates GA, Murphy M, Rees TS, Fraher A. Screnning for handicap hearing loss in the elderly. J Fam Pract 2003; 52:56-62.

2. Sindhusake D, Mitchell P, Smith W, Golding M, Newall P, Hartley D, et al. Validation of self-reported hearing loss. The Blue Mountains Hearing Study. Int J Epidemiol 2001; 30:1371-8.

3. Zhan W, Cruickshanks KJ, Klein BE, Klein R, Huang GH, Pankow JS, et al. Generation differences in the prevalence of hearing impairment in older adults. Am J Epidemiol 2010; 171:260-6.

4. Gates GA, Cooper JC, Kannel WB, Miller NJ. Hearing in the elderly: The Framingham Cohort, 19831985. Ear Hear 1990; 11:247-56.

5. Davis A. Hearing in adults. The prevalence and distribution of hearing impairment and reported hearing disability in the MRC Institute of Hearing Research's National Study of Hearing. MRC Institute of Hearing Research. London: Whurr Publishers; 1995.
6. Mattos LC, Veras RP. Prevalência da perda auditiva em uma população de idosos da cidade do Rio de Janeiro: um estudo seccional. Rev Bras Otorrinolaringol 2007; 73:654-9.

7. Viúde A. Fatores associados à presbiacusia em idosos [Dissertação de Mestrado]. São Paulo: Universidade de São Paulo; 2002.

8. Khabori MA, Khandekar R. The prevalence and causes of hearing impairment in Oman: a community-based cross-sectional study. Int J Audiol 2004; 43:486-92.

9. Secretaria de Atenção à Saúde, Ministério da Saúde. Política Nacional de Atenção à Pessoa Portadora de Deficiência. Brasília: Ministério da Saúde; 2002.

10. Gatehouse S. Benefits of and candidature for hearing aid features the audiogram is not enough. In: Proceedings of the 1st International Congress on Geriatric/Gerontologic Audiology. Stockholm: Linköping University; 2004. p. 12-5. 
11. Idrizbegovic E. The effects of ageing in the peripheral and central auditory system. In: Proceedings of the 1st International Congress on Geriatric/Gerontologic Audiology. Stockholm: Linköping University; 2004. p. 6-9.

12. Nondahl DM, Cruickshanks KJ, Wiley TL, Tweed TS, Klein R, Klein BEK. Accuracy of self-report hearing loss. Int J Audiol 1998; 37:295-301.

13. Torre III P, Moyer CJ, Haro NR. The accuracy of self-report hearing loss in older Latino-American adults. Int J Audiol 2006; 45:559-62.

14. Hashimoto H, Nomura K, Yano E. Psychosomatic status affects the relationship between subjective hearing difficulties and the results of audiometry. J Clin Epidemiol 2004; 57:381-5.

15. Pascolini D, Smith A. Hearing impairment in 2008: a compilation of available epidemiological studies. Int J Audiol 2009; 48:473-85.

16. Cesar CLG, Carandina L, Alves MCGP, Barros MBA, Goldbaum M. Saúde e condição de vida em São Paulo: inquérito multicêntrico de saúde no Estado de São Paulo - ISA-SP. São Paulo: Faculdade de Saúde Pública, Universidade de São Paulo; 2005.

17. Cesar CLG, Segri NJ, Sportello R. Inquéritos de saúde. São Paulo: Faculdade de Saúde Pública; 2003 http://www.fsp.usp.br/isa-sp/index_arquivos/Pa ge3157.htm (acessado em 15/Fev/2011).

18. Lima-Costa MF, Barreto SM, Giatti L. Condições de saúde, capacidade funcional, uso de serviços de saúde e gastos com medicamentos da população idosa brasileira: um estudo descritivo baseado na Pesquisa Nacional por Amostra de Domicílios. Cad Saúde Pública 2003; 19:735-43.

19. Castro SS, Cesar CLG, Carandina L, Barros MBA, Alves MCGP, Goldbaum M. Deficiência visual, auditiva e física: prevalência e fatores associados em estudo de base populacional. Cad Saúde Pública 2008; 24:1773-82.

20. Rosenhall U. Ageing in the auditory and vestibular systems. In: Luxon L, Furman JM, Martini A, Stephens SDG, editors. Textbook of audiological medicine: clinical aspects of hearing and balance. London: Martin Dunitz; 2003. p. 13-24.

21. Wilson DH, Walsh PG, Sanchez L, Davis AC, Taylor AW, Tucker G, et al. The epidemiology of hearing impairment in an Australian adult population. Int J Epidemiol 1999; 28:247-52.

22. Cruickshanks KJ, Tweed TS, Wiley TL, Klein BEK, Klein R, Chappell R, et al. The 5-year incidence and progression of hearing loss: the epidemiology of hearing loss study. Arch Otolaryngol Head Neck Surg 2003; 129:1041-6.
23. Fook L, Morgan R. Hearing impairment in older people: a review. Postgrad Med J 2000; 76:537-41.

24. Morettin M, Cardoso MRA, Lebrão ML, Duarte YAO. Fatores relacionados à autopercepção da audição entre idosos do Município de São Paulo Projeto SABE. Saúde Coletiva 2008; 5:168-72.

25. Greco MC, Russo ICP. Achados audiológicos em indivíduos idosos em uma clínica particular de São Paulo - SP. Acta ORL 2006; 24:45-54.

26. Baraldi GS, Almeida LC, Borges ACC. Evolução da perda auditiva no decorrer do envelhecimento. Rev Bras Otorrinolaringol 2007; 73:64-70.

27. Mazelova J, Popelar J, Syka J. Auditory function in presbycusis: peripheral X central changes. Exp Gerontol 2003; 38:87-94.

28. Russo ICP. Intervenção audiológica no idoso. In: Ferreira LP, Befi-Lopes DM, Limongi SCO, organizadores. Tratado de fonoaudiologia. São Paulo: Roca; 2004. p. 585-96.

29. Teixeira AR, Freitas CR, Millão LF, Gonçalves AK, Becker Junior B, Santos AMPV, et al. Relação entre a queixa e a presença de perda auditiva entre idosos. Arq Int Otorrinolaringol (Impr) 2009; 13:78-82.

30. Fernandez HM. Vision and hearing impairment. In: Cassel HJ, Larson EB, Meier DE, editors. Geriatric medicine: an evidence based approach. $4^{\text {th }} \mathrm{Ed}$. New York: Springer; 2003. p. 143-63.

31. Bagai A, Thavendiranathan P, Detsky AS. Does this patient have hearing impairment? JAMA 2006; 295:416-28.

32. Cruz MS, Oliveira LR, Carandina L, Lima MCP, Cesar CLG, Barros MBA, et al. Prevalência de deficiência auditiva referida e causas atribuídas: um estudo de base populacional. Cad Saúde Pública 2009; 25:1123-31.

33. Brasil. Lei ${ }^{\circ}$. 8842, de 04 de janeiro de 1994. Dispõe sobre a política nacional do idoso, cria o Conselho nacional do Idoso e dá outras providências. Diário Oficial da União 1994; 4 jan.

34. Marques ACO, Kozlowski L, Marques JM. Reabilitação auditiva no idoso. Rev Bras Otorrinolaringol 2004; 70:806-11.

35. Ministério da Saúde. Portaria GM/MS nº. 2.073 de 28 de setembro de 2004. Institui a Política Nacional de Atenção à Saúde Auditiva. Diário Oficial da União 2004; 28 set.

Recebido em 19/Nov/2010

Versão final reapresentada em 21/Fev/2011

Aprovado em 06/Mai/2011 